\title{
Bushen Culuan Decoction
}

National Cancer Institute

\section{Source}

National Cancer Institute. Bushen Culuan Decoction. NCI Thesaurus. Code C160712.

A traditional Chinese medicine (TCM) decoction containing a mixture of ten Chinese herbs including Tusizi, Yinyanghuo, Xianmao, Xuduan, Gouqizi, Nvzhenzi, Zelan, Sheng puhuang, Xiangfu and Chuanshanlong, with potential to induce ovulation. Upon oral administration, Bushen Culuan decoction may, through a not yet fully elucidated mechanism, depress follicle-stimulating hormone (FSH) levels, elevate anti-Mullerian hormone (AMH) levels, and increase the number of antral follicle counts (AFCs), thereby promoting ovulation. 